\title{
Ulcerative colitis - what can we learn from the Asia-Pacific region?
}

\author{
Subrata Ghosh MBBS MD FRCPC FRCP FRCPE, Editor-in-Chief
}

A recent epidemiological study clearly demonstrated that inflammatory bowel disease (IBD), especially ulcerative colitis, is a rapidly emerging disease in the Asia Pacific region (1). A systematic review of the global incidence and prevalence of IBD before publication of the Asia Pacific data (2) confirmed the worldwide nature of IBD. In timetrend analyses, it showed that $75 \%$ of Crohn disease studies and $60 \%$ of ulcerative colitis studies reported statistically significant increases in incidence (2). The emergence of IBD in the Asia Pacific region suggests strong environmental influence on the pathogenesis of IBD including socioeconomic, lifestyle and dietary changes.

The age-standardized incidence of ulcerative colitis in the Asia Pacific region ranged from 0.24 to 7.47 per 100,000 population, with the lowest incidence in Bangkok and the highest in Australia. Even highly westernized Asian countries, such as Hong Kong, have an ulcerative colitis incidence of 1.30 per 100,000 population (1). The incidence of Crohn disease in Australia was nearly double that of ulcerative colitis; however, in the Asian countries, ulcerative colitis was generally more prevalent than Crohn disease. In a recent Asia Pacific epidemiology study of IBD incidence (1), the distribution of ulcerative colitis location in Australia and in Asia were similar, with approximately one-third each being proctitis, left-sided colitis, and extensive or total colitis (1). The Asia Pacific epidemiology study did not include South Korea, Japan or India, which tend to have a higher incidence of IBD than other Asian countries. However, in the current issue of the Journal, Park et al (3) (pages 125 to 130) reported a retrospective analysis of disease location at diagnosis in 240 ulcerative colitis patients diagnosed at Asan Medical Centre, Seoul, South Korea. Approximately 19\% of patients had an atypical distribution of disease, including patchy or skip lesions or, less commonly, rectal sparing. Such atypical distribution was more common in patients reported to demonstrate appendiceal orifice inflammation. Skip lesions were more common in the proximal colon. While such atypical distribution inevitably raises the question of Crohn's colitis, $67 \%$ of ulcerative colitis patients showing atypical distribution at diagnosis had reverted to the typical distribution of lesions at follow-up colonoscopy. The ulcerative colitis disease course and outcome of individuals with atypical disease location was similar to those with more classical disease location.

While such atypical distribution of ulcerative colitis has been sporadically reported in the West $(4,5)$, including $27 \%$ of ulcerative colitis patients showing discontinuous inflammation of the appendiceal orifice in a prospective study, rectal sparing is considered to be unusual in the West after meticulous pathological examination of colectomy specimens (6). This is not surprising given that in the study by Park et al (3), follow-up colonoscopy showed that the distribution of ulcerative colitis had reverted to the classical in the majority of patients. However, several reports from Asia, especially Japan, Korea and India, with higher incidence of ulcerative colitis in Asia, have reported atypical distribution of ulcerative colitis (7-9) despite the contrary data in the recent Asia Pacific epidemiology study. Although single-centre and retrospective, the study by Park et al (3) substantiates these previous findings. It is important to recognize that the Asia Pacific region is a vast, inhomogeneous area with multiple cultures, lifestyles, environment, diet and genetics. For example, three regions of mainland China had ulcerative colitis incidences of 2.05, 0.42 and 0.41 per 100,000 population (1), suggesting unique environmental influences in specific regions.

The study by Park et al (3) and previous studies encourage us to obtain additional epidemiological and environmental data collected prospectively from geographical regions with emerging IBD. It will be important to understand whether disease presentation varies in different geographical areas based on colonic microbiome, dietary habits and genetics. This may shed light on its pathogenesis and provide more evidence to answer the question whether ulcerative colitis is 'one disease or many'. This discussion may be even more relevant in the case of Crohn disease. It especially points to the challenges of epidemiological studies that attempt to distinguish between ulcerative colitis and Crohn's colitis.

\section{REFERENCES}

1. Ng SC, Tang W, Ching JY, et al. Incidence and phenotype of inflammatory bowel disease based on results from the Asia-pacific Crohn's and colitis epidemiology study. Gastroenterology 2013;145:158-65.

2. Molodecky NA, Soon IS, Rabi DM, et al. Increasing incidence and prevalence of the inflammatory bowel diseases with time, based on systematic review. Gastroenterology 2012;142:46-54.

3. Park SH, Yang S-K, Park S-K, et al. Atypical distribution of inflammation in newly diagnosed ulcerative colitis is not rare. Can J Gastroenterol Hepatol 2014;28:125-30.

4. Ladefoged K, Munck LK, Jorgensen F, Engel P. Skip inflammation of the appendiceal orifice: A prospective endoscopic study. Scand J Gastroenterol 2005;40:1192-6.

5. Hill MD, Davies G, McIntyre AS, Gorard DA. Proctitis with caecitis: An atypical presentation of ulcerative colitis. Endoscopy 2002;34:664-6.

6. Joo M, Odze RD. Rectal sparing and skip lesions in ulcerative colitis: A comparative study of endoscopic and histologic findings in patients who underwent proctocolectomy. Am J Surg Pathol 2010;34:689-96.

7. Hokama A, Ihama Y, Chinen H, Kishimoto K, Kinjo F, Fujita J. Appendiceal orifice inflammation in ulcerative colitis. Dig Dis Sci 2010;55:1189.

8. Shah SN, Amarapurkar AD, Shrinivas N, Rathi PM. Atypical histological features of ulcerative colitis. Trop Gastroenterology 2011;32:107-11.

9. Sood A, Midha V, Sood N, Kaushal V. Skip lesions in ulcerative colitis. Indian J Gastroenterol 2000;19:143. 


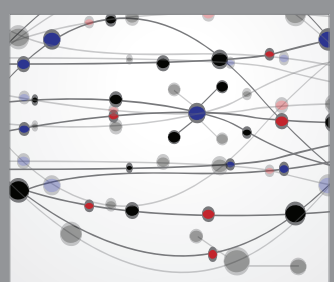

The Scientific World Journal
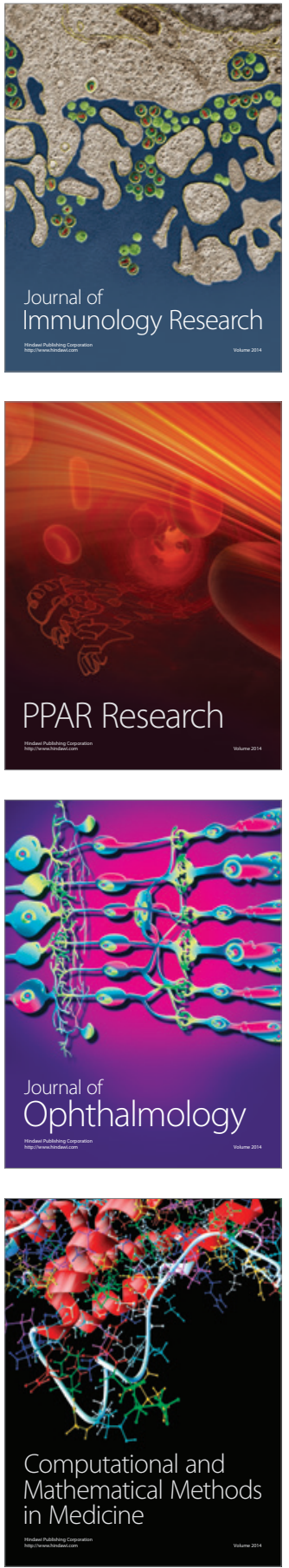

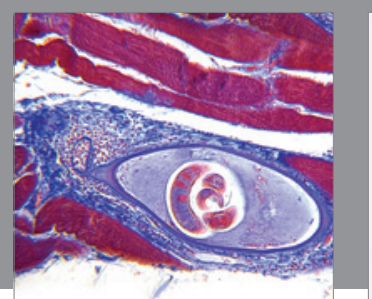

Gastroenterology Research and Practice

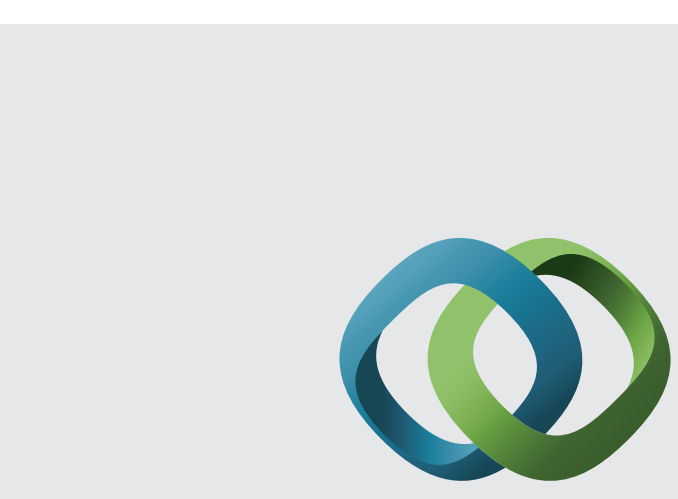

\section{Hindawi}

Submit your manuscripts at

http://www.hindawi.com
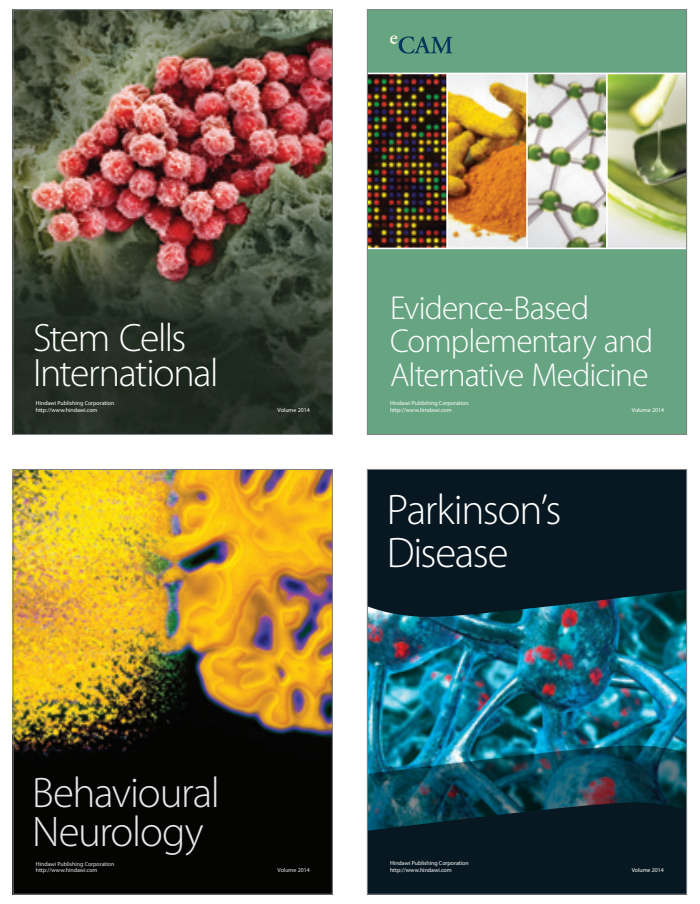
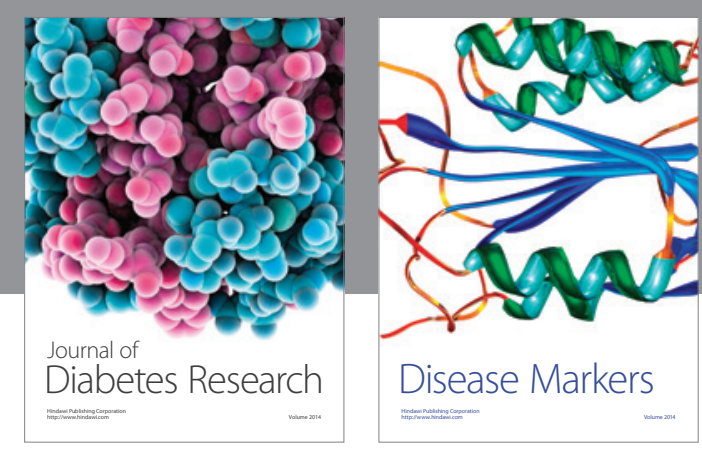

Disease Markers
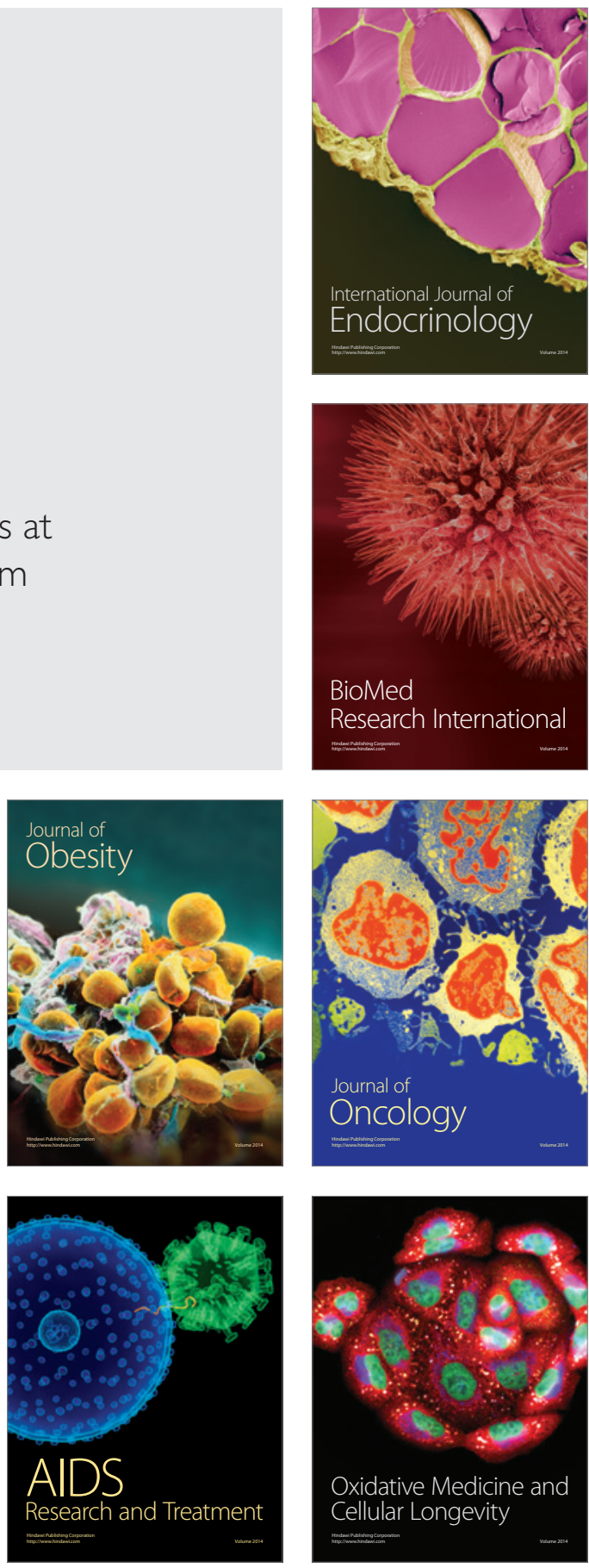